\title{
Reduced dielectric response in spatially varying electric fields.
}

\author{
Hansen, Jesper Schmidt
}

Published in:

Journal of Chemical Physics

DOI:

$10.1063 / 1.4935825$

Publication date:

2015

Document Version

Publisher's PDF, also known as Version of record

Citation for published version (APA):

Hansen, J. S. (2015). Reduced dielectric response in spatially varying electric fields. Journal of Chemical Physics, 143, [194507]. https://doi.org/10.1063/1.4935825

\section{General rights}

Copyright and moral rights for the publications made accessible in the public portal are retained by the authors and/or other copyright owners and it is a condition of accessing publications that users recognise and abide by the legal requirements associated with these rights.

- Users may download and print one copy of any publication from the public portal for the purpose of private study or research.

- You may not further distribute the material or use it for any profit-making activity or commercial gain.

- You may freely distribute the URL identifying the publication in the public portal.

\section{Take down policy}

If you believe that this document breaches copyright please contact rucforsk@kb.dk providing details, and we will remove access to the work immediately and investigate your claim. 


\section{AlP $\mid \begin{aligned} & \text { The Journal of } \\ & \text { Chemical Physics }\end{aligned}$}

\section{Reduced dielectric response in spatially varying electric fields}

\section{J. S. Hansen}

Citation: The Journal of Chemical Physics 143, 194507 (2015); doi: 10.1063/1.4935825

View online: http://dx.doi.org/10.1063/1.4935825

View Table of Contents: http://scitation.aip.org/content/aip/journal/jcp/143/19?ver=pdfcov

Published by the AIP Publishing

\section{Articles you may be interested in}

Electric-field-induced strain contributions in morphotropic phase boundary composition of (Bi1/2Na1/2)TiO3$\mathrm{BaTiO} 3$ during poling

Appl. Phys. Lett. 107, 242902 (2015); 10.1063/1.4937470

Proteins in the electric field near the surface of mica

J. Chem. Phys. 139, 045102 (2013); 10.1063/1.4813854

On the response of large systems to electrostatic fields

AIP Conf. Proc. 1461, 116 (2012); 10.1063/1.4736878

Electric field and spatial charge formation in glasses and glassy nanocomposites

J. Appl. Phys. 109, 094108 (2011); 10.1063/1.3582131

Response of polar nanoregions in $68 \% \mathrm{~Pb}(\mathrm{Mg} 1 / 3 \mathrm{Nb} 2 / 3)$ O 3 - $32 \% \mathrm{~Pb}$ Ti O 3 to a [001] electric field Appl. Phys. Lett. 93, 082901 (2008); 10.1063/1.2959077

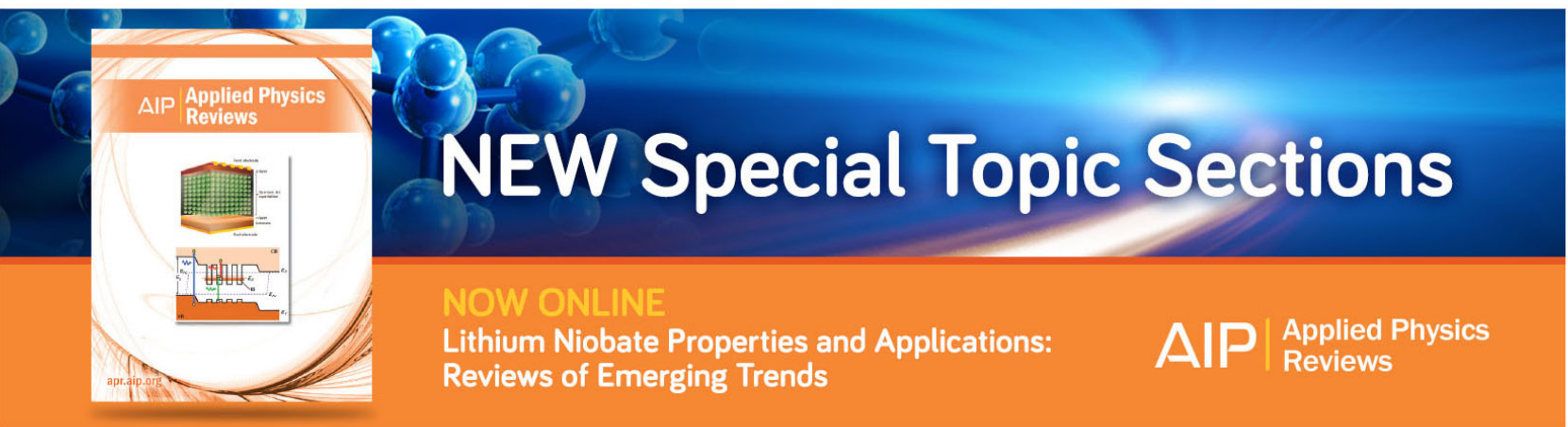




\title{
Reduced dielectric response in spatially varying electric fields
}

\author{
J. S. Hansen a) \\ DNRF Centre “Glass and Time," IMFUFA, Department of Sciences, Roskilde University, P.O. Box 260, \\ Roskilde DK-4000, Denmark
}

(Received 14 August 2015; accepted 2 November 2015; published online 18 November 2015)

\begin{abstract}
In this paper, the dynamical equation for polarization is derived. From this the dielectric response to a spatially varying electric field is analyzed showing a reduced response due to flux of polarization in the material. This flux is modeled as a diffusive process through linear constitutive relations between the flux and the gradient of the polarization. Comparison between the theory and molecular dynamics simulations confirms this effect. The effect is significant for small length scale electric field variations and the inclusion of the flux is thus important in nanoscale modeling of dielectric response. () 2015 AIP Publishing LLC. [http://dx.doi.org/10.1063/1.4935825]
\end{abstract}

\section{INTRODUCTION}

For weak electric fields the dielectric response is commonly modeled by a linear constitutive relation between the polarization and the field. ${ }^{1}$ In the case of a spatially varying field the local response is given by a convolution of the dielectric susceptibility and the electric field. ${ }^{2}$ In Fourier space this corresponds to introducing a wave vector dependent susceptibility, or kernel, which then accounts for the response at different wavelengths.

The fact that there exists a local response means that the polarization of the material can vary, that is, there exists a polarization gradient. This gradient must, intuitively, result in a corresponding flux. To the author's knowledge, this additional mechanism is often overlooked and its effect unwarily incorporated into the susceptibility kernel hiding the actual underlying mechanism. Dahler and Scriven ${ }^{3}$ derived the relevant balance equation, but application of the theory is not shown. de Groot and Suttorp, ${ }^{4}$ de Groot and Mazur, ${ }^{1}$ and more recently Dávalos-Orozco and del Castillo ${ }^{5,6}$ derived the full dynamical equations for polar viscoelastic fluids; however, their approach is based on the Maxwell stress ${ }^{1}$ and different from the one presented here and, therefore, leads to a different formalism.

In this paper, the fundamental balance equation for the polarization is derived from the microscopic hydrodynamic operator (MH-operator). ${ }^{7}$ This particular formalism gives a microscopic interpretation of the polarization flux in terms of the molecular degrees of freedom in the strict limit of zero wave vector. Linear constitutive relations are proposed relating the flux to thermodynamic forces through differential operators acting on the polarization. The theory is then applied to an isotropic linear dielectric in a non-uniform electric field and a dimensionless number quantifying the effect of the flux is defined. Molecular dynamics (MD) simulations are performed for two dielectric materials and data of the dielectric response are analyzed in the framework of the theory.

\section{THEORY}

\section{A. Polarization balance equation}

Consider a dielectric material composed of permanent molecular dipoles. Only the alignment of the dipoles with the field is treated here and induced effects are ignored. Let $\boldsymbol{\mu}_{i}$ be the dipole moment of molecule $i$ with center-of-mass position $\mathbf{r}_{i}$, the polarization (dipole moment density) $\mathbf{P}$ is then defined, up to the dipole moment, ${ }^{4}$ as

$$
\mathbf{P}(\mathbf{r}, t)=\rho(\mathbf{r}, t) \mathbf{p}(\mathbf{r}, t)=\sum_{i} \boldsymbol{\mu}_{i}(t) \delta\left(\mathbf{r}-\mathbf{r}_{i}(t)\right),
$$

where $\rho$ is the mass density, $\mathbf{p}$ is the dipole moment per unit mass, and $\delta$ is the Dirac delta. In Fourier space we have for wave vector $\mathbf{k}$,

$$
\widetilde{\rho \mathbf{p}}(\mathbf{k}, t)=\sum_{i} \boldsymbol{\mu}_{i}(t) e^{-i \mathbf{k} \cdot \mathbf{r}_{i}(t)}
$$

From here on, the explicit wave vector and time dependence are omitted unless they provide valuable information. The rate of change is given by differentiation of Eq. (2),

$$
\frac{\partial}{\partial t} \widetilde{\rho \mathbf{p}}(\mathbf{k}, t)=\sum_{i}\left(\frac{\mathrm{d} \mu_{i}}{\mathrm{~d} t}+\mu_{i}\left(-i \mathbf{k} \cdot \mathbf{v}_{i}\right)\right) e^{-i \mathbf{k} \cdot \mathbf{r}_{i}},
$$

which in the strict limit of $\mathbf{k} \rightarrow \mathbf{0}$ is

$$
\frac{\partial}{\partial t} \widetilde{\rho \mathbf{p}}(\mathbf{k}, t)=\sum_{i}\left(1-i \mathbf{k} \cdot \mathbf{r}_{i}\right) \frac{\mathrm{d} \boldsymbol{\mu}_{i}}{\mathrm{~d} t}-i \mathbf{k} \cdot \sum_{i} \mathbf{v}_{i} \boldsymbol{\mu}_{i}
$$

Note the identity $\boldsymbol{\mu}_{i}\left(-i \mathbf{k} \cdot \mathbf{v}_{i}\right)=-i \mathbf{k} \cdot\left(\mathbf{v}_{i} \boldsymbol{\mu}_{i}\right)$, where $\mathbf{v}_{i} \boldsymbol{\mu}_{i}$ is the dyadic between the molecular velocity $\mathbf{v}_{i}$ and the dipole moment $\boldsymbol{\mu}_{i}$. The resultant is a rank-2 tensor.

The molecular velocity $\mathbf{v}_{i}$ is decomposed into the thermal (or peculiar) velocity, $\mathbf{c}_{i}$, and the streaming velocity, $\mathbf{u}\left(\mathbf{r}_{i}, t\right)$. That is, letting $\mathbf{v}_{i}=\mathbf{c}_{i}+\mathbf{u}\left(\mathbf{r}_{i}, t\right)$ one arrives at, in short-hand notation,

\footnotetext{
a) jschmidt@ ruc.dk
}

$$
\frac{\partial}{\partial t} \widetilde{\rho \mathbf{p}}(\mathbf{k}, t)=\mathcal{H}\left[\boldsymbol{\mu}_{i}\right],
$$


where $\mathcal{H}$ is the $\mathrm{MH}$-operator ${ }^{7}$ acting on the molecular dipole moment

$$
\begin{aligned}
\mathcal{H}\left[\boldsymbol{\mu}_{i}\right]= & \sum_{i}\left(1-i \mathbf{k} \cdot \mathbf{r}_{i}\right) \frac{\mathrm{d} \boldsymbol{\mu}_{i}}{\mathrm{~d} t} \\
& -i \mathbf{k} \cdot \sum_{i} \mathbf{c}_{i} \boldsymbol{\mu}_{i}-i \mathbf{k} \cdot \sum_{i} \mathbf{u}\left(\mathbf{r}_{i}, t\right) \boldsymbol{\mu}_{i} .
\end{aligned}
$$

It is convenient to express the rate of change of the molecular dipole moment according to Euler's equation of motion. For rigid molecules the permanent dipole will be a constant in the molecule's principle frame of reference. The rate of change is then

$$
\frac{\mathrm{d} \boldsymbol{\mu}_{i}}{\mathrm{~d} t}=\boldsymbol{\Omega}_{i} \times \boldsymbol{\mu}_{i},
$$

where $\boldsymbol{\Omega}_{i}$ is the molecular intrinsic angular velocity seen from the inertial frame. Substitution yields the polarization balance equation in Fourier space for $\mathbf{k} \rightarrow 0$,

$$
\begin{aligned}
\frac{\partial}{\partial t} \widetilde{\rho \mathbf{p}}(\mathbf{k}, t)= & -i \mathbf{k} \cdot \sum_{i} \mathbf{u}\left(\mathbf{r}_{i}, t\right) \boldsymbol{\mu}_{i}+\sum_{i} \boldsymbol{\Omega}_{i} \times \boldsymbol{\mu}_{i} \\
& -i \mathbf{k} \cdot\left(\sum_{i} \mathbf{c}_{i} \boldsymbol{\mu}_{i}+\mathbf{r}_{i}\left(\boldsymbol{\Omega}_{i} \times \boldsymbol{\mu}_{i}\right)\right) .
\end{aligned}
$$

In real space this suggests a balance equation in real space at small wave vector

$$
\frac{\partial \rho \mathbf{p}}{\partial t}=-\boldsymbol{\nabla} \cdot(\rho \mathbf{u p})+\frac{2 \rho}{3}(\boldsymbol{\Omega} \times \boldsymbol{\mu})-\boldsymbol{\nabla} \cdot \mathbf{R} .
$$

Except for the factor 2/3, this is in agreement with the result derived by Dahler and Scriven. ${ }^{3}$ Each term on the right-hand side is discussed in the following. The first term, i.e., the advection, is

$$
\rho \mathbf{u p}=\mathbf{u} \rho \mathbf{p}=\frac{1}{V}\left\langle\sum_{i} \mathbf{u}\left(\mathbf{r}_{i}, t\right) \sum \boldsymbol{\mu}_{i}\right\rangle=\frac{1}{V}\left\langle\sum_{i} \mathbf{u}\left(\mathbf{r}_{i}, t\right) \boldsymbol{\mu}_{i}\right\rangle
$$

as the cross correlation effects are assumed to be zero, that is,

$$
\left\langle\sum_{i} \sum_{i \neq j} \mathbf{u}\left(\mathbf{r}_{i}, t\right) \boldsymbol{\mu}_{j}\right\rangle=\mathbf{0} .
$$

Importantly, one sees that the ensemble average is taken of the product (this case the dyadic). The product of the ensemble averages will lead to an inconsistency with the microscopic derivation. For the second term on the right-hand side in Eq. (9) it is noted that $\boldsymbol{\Omega}=\frac{3}{2}\left\langle\sum_{i} \boldsymbol{\Omega}_{i}\right\rangle{ }^{8}$ We then have

$$
\boldsymbol{\Omega} \times \rho \mathbf{p}=\frac{1}{V}\left\langle\frac{3}{2} \sum_{i} \boldsymbol{\Omega}_{i} \times \sum_{i} \boldsymbol{\mu}_{i}\right\rangle=\frac{3}{2 V}\left\langle\sum_{i} \boldsymbol{\Omega}_{i} \times \boldsymbol{\mu}_{i}\right\rangle,
$$

where, again, the cross correlation in Eq. (8) effects are zero. Notice, the factor $3 / 2$. Finally, the last term defines a polarization flux tensor for zero wave vector, that is,

$$
\mathbf{R}(t)=\frac{1}{V}\left\langle\sum_{i} \mathbf{c}_{i} \boldsymbol{\mu}_{i}+\mathbf{r}_{i}\left(\boldsymbol{\Omega}_{i} \times \boldsymbol{\mu}_{i}\right)\right\rangle .
$$

This definition is analogous to the Irving-Kirkwood interpretation. $^{9}$
The second term on right-hand side of Eq. (9) can be regarded as a production term and is non-zero if the dipoles are rotating around their center of mass. Non-zero rotation can be achieved by a rotating electric field. ${ }^{10,11}$ Due to the production term, the polarization is not a conserved quantity but couples to the fluid's mechanical properties. From the microscopic definition it is also seen that the polarization flux tensor $\mathbf{R}$ is non-symmetric as both dyadics, $\mathbf{c p}$ and $\mathbf{r}(\mathbf{\Omega} \times \mathbf{p})$, are non-symmetric.

\section{B. Local constitutive relations}

If one follows the usual decomposition, ${ }^{12,13}$ the polarization flux tensor can be written as a sum of the trace, $R$, traceless symmetric part, $\mathbf{R}$, and the antisymmetric part $\stackrel{a}{\mathbf{R}}$. That is, $\mathbf{R}=R \mathbf{I}+\stackrel{\text { os }}{\mathbf{R}}+\stackrel{a}{\mathbf{R}}$. $\mathbf{I}$ is the unit tensor. Here the anti-symmetric tensor is given by its (pseudo) vector dual $\stackrel{a d}{\mathbf{R}}=\left(\stackrel{a}{R_{y z}}, \stackrel{a}{{ }_{z x}}, \stackrel{a}{R_{x y}}\right)$. For linear isotropic dielectrics these three fluxes are proposed to be associated with the divergence, gradient, and curl of polarization field through the linear phenomenological relations

$$
R=-\chi_{v}(\boldsymbol{\nabla} \cdot \mathbf{p}), \quad \stackrel{o s}{\mathbf{R}}=-2 \chi_{0}(\stackrel{o s}{\nabla \mathbf{p}}),
$$

and

$$
\stackrel{a d}{\mathbf{R}}=-\chi_{r}(\boldsymbol{\nabla} \times \mathbf{p}) .
$$

Here $\chi_{v}, \chi_{0}$, and $\chi_{r}$ are phenomenological transport coefficients. Note that the constitutive relations, Eq. (14), are local in that the flux is only dependent on the local derivatives of $\mathbf{p}$.

Allowing for an external forcing term, $\sigma_{P}$, and substituting Eq. (14) into Eq. (9) one arrives at

$\frac{\partial \rho \mathbf{p}}{\partial t}+\boldsymbol{\nabla} \cdot(\rho \mathbf{u p})=\boldsymbol{\sigma}_{P}+\frac{2 \rho}{3}(\mathbf{\Omega} \times \mathbf{p})+\chi_{l} \boldsymbol{\nabla}(\boldsymbol{\nabla} \cdot \mathbf{p})+\chi_{t} \nabla^{2} \mathbf{p}$,

with $\chi_{t}=\chi_{0}+\chi_{r}$ and $\chi_{l}=\chi_{v}+\chi_{0} / 3-\chi_{r}$. Equation (15) is the dynamical equation for the polarization in the hydrodynamic regime of small wave vector.

\section{Application of a sinusoidal electrical field}

Let an external electric field act on a dielectric material. The field is sinusoidal varying in the $z$-direction and acting in the $x$-direction only,

$$
\mathbf{E}^{\mathrm{ext}}(z)=E_{0} \sin \left(k_{n} z\right) \mathbf{i},
$$

where $E_{0}$ is the field amplitude, $k_{n}=2 \pi n / L$ is the field wave vector, $n$ is the wave number, and $\mathbf{i}$ is the unit vector parallel to the $x$-axis. $L$ is the length of the system in the $z$-direction.

The following two assumptions are made: (i) The local electric field is given by $\mathbf{E}^{\text {ext }}$, that is, the field due to the polarization is negligible. This is justified for small molecular dipole moments; in fact, this approximation is applied even for bulk water in the presence of an external field. ${ }^{14}$ (ii) According to Faraday's law, $\dot{\mathbf{B}}=-\boldsymbol{\nabla} \times \mathbf{E}$, the electric field is associated with a magnetic field $\mathbf{B}(t)=E_{0} k_{n} \cos \left(k_{n} z\right) t \mathbf{j}$, 
where $\mathbf{j}$ is the unit vector parallel to the $y$-axis. The amplitude of the magnetic field is therefore linearly increasing in time, and the induced magnetic field can only be ignored in some time interval $t<\tau^{\prime}$ such that $E_{0} k_{n} \tau^{\prime}<B_{0}$, where $B_{0}$ is the critical magnetic field strength. Furthermore, here only the steady state is studied, that is, for times larger than the dipole relaxation time $\tau$. This means that the time interval is limited to $\tau<t<\tau^{\prime}$. The second assumption is that such time interval exists.

For linear dielectrics the polarization at a given point is given by $p_{x}=\kappa E_{x}^{\mathrm{ext}}$, where subscript $x$ indicates vector $x$-component, and $\kappa$ is the dielectric susceptibility per unit mass. $K$ is here in the local description assumed to be wave vector independent. The external forcing can now be written as $^{1}$

$$
\sigma_{\mathbf{P}}=\frac{\rho}{\tau}\left(\kappa E_{x}^{\mathrm{ext}}-p_{x}\right)
$$

In steady state Eq. (15) reduces to a simple two point boundary value problem,

$$
\frac{1}{\tau}\left(\kappa E_{x}^{\mathrm{ext}}-p_{x}\right)+v_{t} \frac{\mathrm{d}^{2} p_{x}}{\mathrm{~d} z^{2}}=0,
$$

with

$$
p_{x}(0)=p_{x}(L)=0
$$

and $v_{t}=\chi_{t} / \rho$. The solution is

$$
p_{x}(z)=\frac{\kappa E_{0} \beta}{k_{n}^{2}+\beta} \sin \left(k_{n} z\right)=A_{n} \sin \left(k_{n} z\right),
$$

where $\beta=1 /\left(v_{t} \tau\right)$ such that $1 / \sqrt{\beta}=\sqrt{v_{t} \tau}$ is a characteristic length scale. From this the dimensionless number, $J e \geq 0$, is introduced,

$$
J e=k_{n}^{2} / \beta=k_{n}^{2} v_{t} \tau .
$$

For small wave vectors where $J e \approx 0$ there is a negligible effect from the flux, that is,

$$
p_{x}(z) \approx \kappa E_{0} \sin \left(k_{n} z\right)=A_{0} \sin \left(k_{n} z\right) .
$$

Note, $A_{0}>A_{n}$ for $n>0$, and that the relative polarization amplitude can be written in terms of $J e$,

$$
\frac{A_{0}}{A_{n}}-1=J e .
$$

\section{MOLECULAR DYNAMICS: METHOD AND RESULTS}

Two dielectric materials are studied using MD simulations. Material I is a generic dielectric where each dipole is a simple diatomic molecule with atomic charges $\pm q$. The atoms not belonging to the same molecule interact through the Weeks-Chandler-Andersen potential ${ }^{15}$ and the Coulomb potential. The intra-molecular force is given by a spring force with zero-force bond length of $1 \sigma$ and spring constant $10^{3} \mathrm{~m}^{2} \sigma^{2} / \epsilon$, where $\sigma, m$, and $\epsilon$ are units of length, mass, and energy, respectively. The state point is $(\rho, T)=\left(0.5 \mathrm{~m} / \sigma^{3}, 1.0 k_{B} / \epsilon\right)$. Material II is water at high temperature $T=508 \mathrm{~K}$ and low density $\rho=33.68 \mathrm{~kg} / \mathrm{m}^{3}$. This state point is chosen as the dielectric response is linear even for very large fields. Also, the relaxation time $\tau$ is small which is convenient from a computational point of view. The water model is the flexible SPC/Fw model, see Refs. 16-18 for details. It is worth stressing that the pressure is positive for both systems. From here on the results for material I are expressed in terms of reduced MD units indicated with a superscript asterisk.

One direct way to implement the electric field is to apply a pulsating field which is turned on in time interval $0<t<\tau^{\prime}$ and then switched off for a time $t>\tau$. One can then sample for $\tau<t<\tau^{\prime}$. To compute the exact time interval the equations of motion for the atoms should be accompanied with simultaneous solution to the Maxwell equations. ${ }^{19}$ In accordance with theory, it is assumed that this interval exists and a fictitious electric field (FEF) is applied which is not associated with a magnetic field. The equations of motion for atom $i$ with mass $m_{i}$, charge $q_{i}$, and position $\mathbf{r}_{i}$ are then $m_{i} \ddot{\mathbf{r}}_{i}=\mathbf{F}_{i}+q_{i} \mathbf{E}^{\text {ext }} . \mathbf{F}_{i}$ is the sum of forces on $i$ due to bond, angle, and pair interactions including Coulomb interactions. It has been shown that for non-confined bulk MD simulations the electrostatic force calculations can be simplified considerably by using shifted force method ${ }^{20}$ and is used here. To control the temperature the system is thermostated using an Andersen-type thermostat. ${ }^{21}$

First, the dipole relaxation time $\tau$ and the dielectric susceptibility per unit mass $\kappa$ is evaluated for zero wave vector by application of a uniform electric field with magnitude $E_{0}$. The total dipole moment per unit mass is calculated from $p_{x}(t)=\left\langle\sum_{i} \mu_{i, x}(t)\right\rangle / \sum_{i} M_{i}$, where $M_{i}$ is the molecular mass. This is fitted to the solution of the homogeneous problem $\dot{p}_{x}=\left(\kappa E_{0}-p_{x}\right) / \tau$, that is,

$$
p_{x}(t)=\kappa E_{0}\left(1-e^{-t / \tau}\right) .
$$

Strictly, the coupling to the angular velocity is neglected here. This fitting is done for varying electric field strengths $E_{0}$; the result for water (material II) is plotted in Fig. 1. For convenience the dipole moment is plotted in the inset rather than the polarization per unit mass. From the inset, it is seen that the dipole relaxation is ballistic for very short times and the fit exclude this region. The linear regime is for

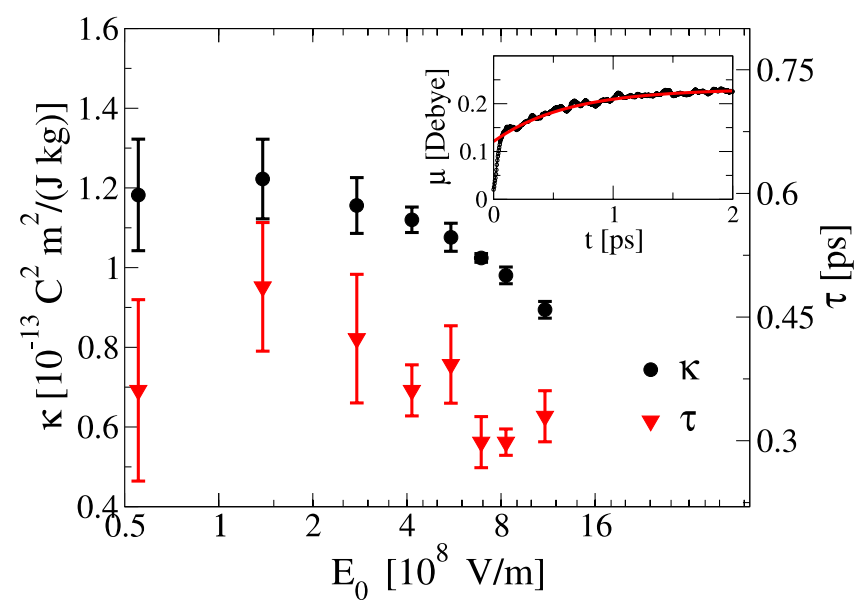

FIG. 1. Dielectric susceptibility per unit mass, $\kappa$, and dipole relaxation time, $\tau$, as a function of field strength $E_{0}$ for water at $(T, \rho)$ $=\left(33.68 \mathrm{~kg} / \mathrm{m}^{3}, 508 \mathrm{~K}\right)$. Inset: Black circles are MD data points and the full line is best fit to Eq. (24) using data points after the ballistic regime. 
$E_{0}<4 \times 10^{8} \mathrm{~V} / \mathrm{m}$ where $\kappa=1.2 \times 10^{-13} \mathrm{C}^{2} \mathrm{~m}^{2} /(\mathrm{J} \mathrm{kg})$. The dipole relaxation time is 0.4 ps. For material I with $q^{*}=1$ the response is linear for all external field strengths studied, $E_{0}^{*} \leq 4$, giving $\kappa^{*}=0.17$ and $\tau^{*}=1.1$.

For the sinusoidal field the local dipole moment per unit mass is evaluated from

$$
p_{x}(z)=\frac{\left\langle\sum_{i} \mu_{i, x} \delta\left(z-z_{i}\right)\right\rangle}{\left\langle\sum_{i} M_{i} \delta\left(z-z_{i}\right)\right\rangle} .
$$

Figure 2(a) shows $p_{x}^{*}$ for material I for $n=4, q^{*}=1$, and $E_{0}^{*}$ $=1$. One clearly sees that the amplitude is reduced compared to the predictions from Eq. (22) with $A_{0}^{*}=\kappa^{*} E_{0}^{*}=0.17$. Equation (20) is also plotted using $v_{t}^{*}$ as the only fitting parameter giving $v_{t}^{*}=0.11$. This value is used to compare the theory with MD data for wave numbers $n=1$ and $n=8$ in Fig. 2(b) showing excellent agreement.

To account for the reduced response one can introduce a wave vector dependent susceptibility, ${ }^{2}$ i.e., the polarization is then given by $p_{x}(z)=\kappa\left(k_{n}\right) E_{0} \sin \left(k_{n} z\right)$. However, according to the theory above the reduction can be due to the additional polarization flux. In that case we should have a diffusion-type dependence, that is, $J e=\kappa E_{0} / A_{n}-1 \propto k_{n}^{2}$, where $A_{n}$ is found
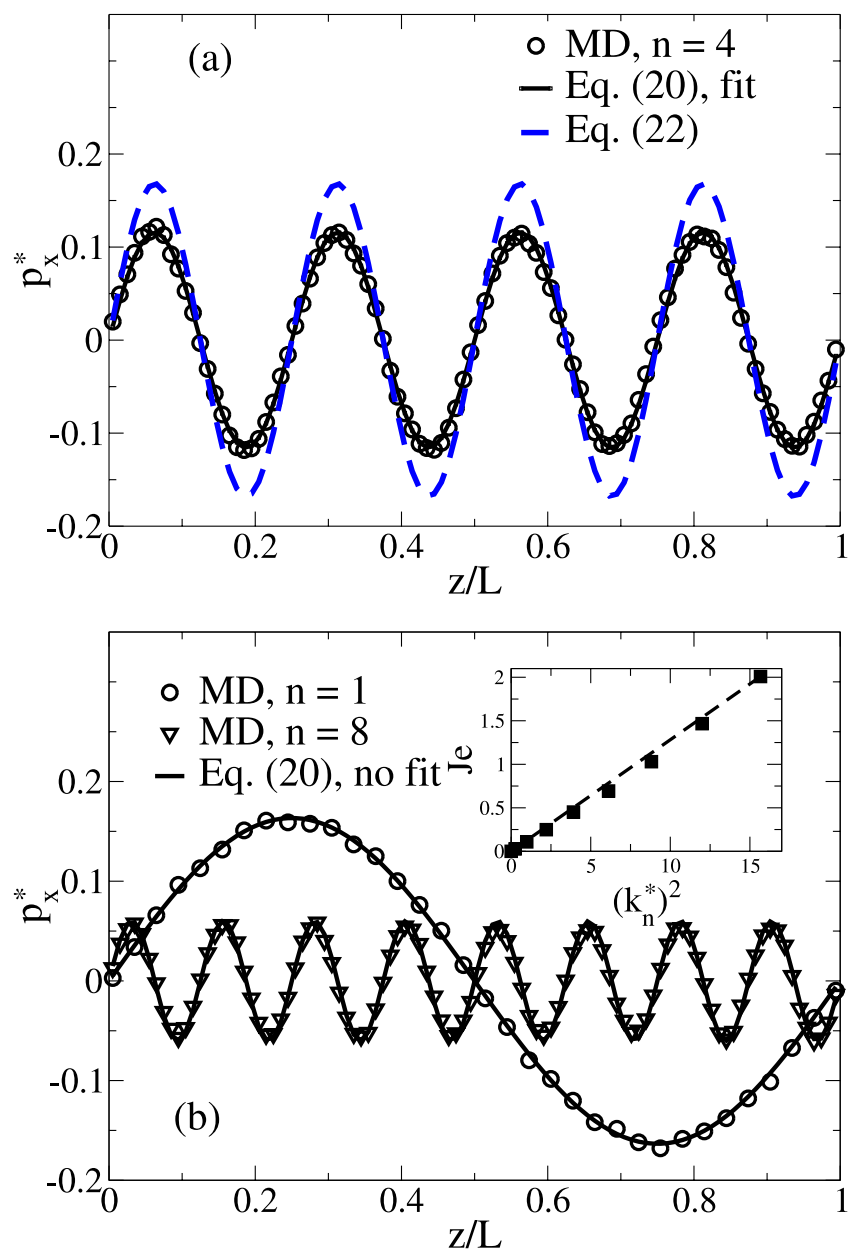

FIG. 2. Local dipole moment per unit mass for material I for $q^{*}=1$ and $E_{0}^{*}=1$. (a) Symbols are MD data, broken line predictions from Eq. (22), and full line is the best fit of Eq. (20) yielding $v_{t}^{*}=0.11$. (b) Comparison between theory, Eq. (20), using $v_{t^{*}}=0.11$, and MD simulations data for two different wave numbers $n=1$ and $n=8$. No fitting is carried out. Inset: Wave vector dependence of Je. Error bars are the size of the symbols.

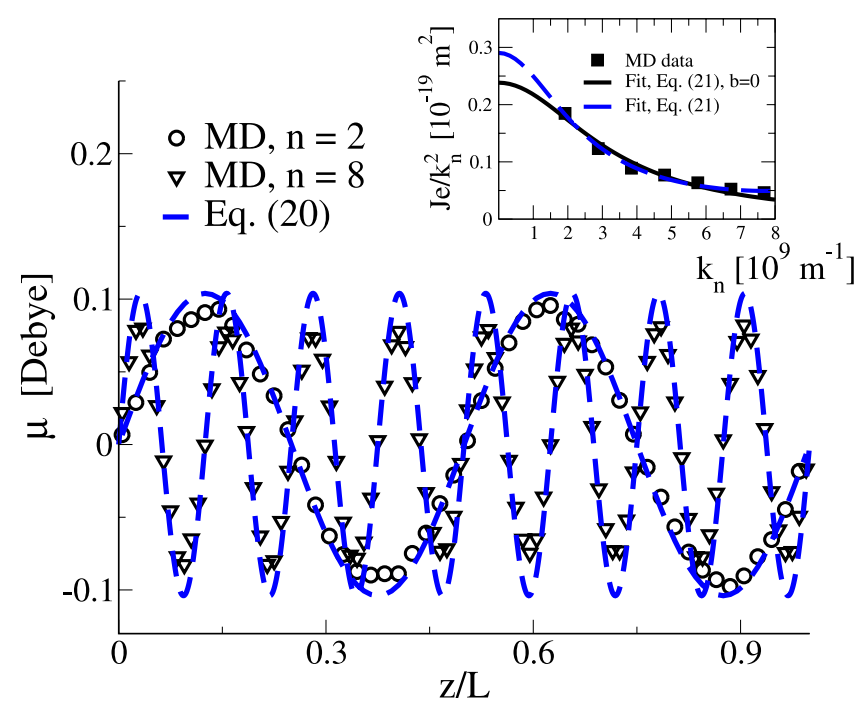

FIG. 3. Local dipole moment for material II (water). Symbols represent MD data and full lines the predictions from Eq. (22). Inset shows the wave vector dependence of $J e / k_{n}^{2}$ : Symbols are MD data and lines are best fit to Eq. (26).

from fitting the MD data to a sine function. The result is shown in the inset of Fig. 2(b), where the wave vector dependence predicted from the theory is confirmed.

The reduced response for large wave vectors is also observed for material II (water), see Fig. 3.

For water $J e$ is not proportional to $k_{n}^{2}$ which indicates that the reduction is not only due to the flux but that the parameters are wave vector dependent in the regime studied here. It is still possible to extract the zero wave vector transport coefficient $v_{t}$. To this end the MD data are fitted to the following expression: ${ }^{22}$

$$
v_{t}\left(k_{n}\right) \tau\left(k_{n}\right)=J e / k_{n}^{2}=\frac{v_{t}\left(k_{n}=0\right) \tau\left(k_{n}=0\right)}{1+a k_{n}^{2}+b k_{n}^{4}}
$$

using $b=0$ (full line) or $b \neq 0$ (broken line). From Fig. 1 the zero wave vector relaxation time $\tau\left(k_{n}=0\right)$ was found to $0.4 \mathrm{ps}$, that is, $v_{t}\left(k_{n}=0\right) \approx 6.5 \times 10^{-8} \mathrm{~m}^{2} / \mathrm{s}$. The effect of the flux is then given by $J e=k_{n}^{2} 2.6 \times 10^{-20} \mathrm{~m}^{2}$, e.g., on a length scale of $3 \mathrm{~nm}$ the flux results in approximately $10 \%$ response reduction according to the local theory.

\section{CONCLUSION}

It was shown that the dielectric response for dielectric materials is reduced on small length scales. The reduction was quantified by the dimensionless number Je. For the generic dumbbell model (material I) the reduction was proportional to the wave vector squared, inset of Fig. 2(b). This is a fingerprint of a diffusive process in accordance with the theory developed in Sec. II. For material II (water) this quadratic dependency was not observed at the state point and wave vectors studied here. This is likely due to the very different molecular structures and corresponding dynamics which can be modeled phenomenologically using wave-vector dependent transport coefficients. 


\section{ACKNOWLEDGMENTS}

The author wish to acknowledge Lundbeckfonden for supporting this work as part Grant No. R49-A5634. The centre for viscous liquid dynamics "Glass and Time" is sponsored by the Danish National Research Foundation from grant DNRF61. The author also thanks Nicholas Bailey and Jeppe Dyre for their comments.

${ }^{1}$ S. R. de Groot and P. Mazur, Non-equilibrium Thermodynamics (Dover Publications, 1984).

${ }^{2}$ J. P. Hansen and I. R. McDonald, Theory of Simple Liquids (Academic Press, Amsterdam, 2006)

${ }^{3}$ J. S. Dahler and L. E. Scriven, "Theory of structured continua I. General considerations of angular momentum and polarization,' Proc. R. Soc. A 27, 504 (1963).

${ }^{4}$ S. R. de Groot and L. G. Suttorp, Foundations of Electrodynamics (NorthHolland Publishing, Amsterdam, 1972).

${ }^{5}$ L. F. del Castillo and L. A. Dávalos-Orozco, "Dielectric relaxation in polar and viscoelastic fluids," J. Chem. Phys. 93, 5147 (1990).

${ }^{6}$ L. A. Dávalos-Orozco and L. F. del Castillo, "Dielectric relaxation in polar and viscoelastic fluids with internal rotation," J. Chem. Phys. 96, 9102 (1992).

${ }^{7}$ J. S. Hansen, J. C. Dyre, P. J. Daivis, B. D. Todd, and H. Bruus, "Continuum nanofluidics," Langmuir (published online).

${ }^{8}$ J. Delhommelle and D. J. Evans, "Poiseuille flow of a micropolar fluid," Mol. Phys. 100, 2857-2865 (2002).

${ }^{9}$ J. H. Irving and J. G. Kirkwood, "The statistical mechanical theory of transport processes. IV. The equations of hydrodynamics," J. Chem. Phys. 18, 817-829 (1950).
${ }^{10}$ J. D. Bonthuis, D. Horinek, L. Bocquet, and R. R. Netz, "Electrohydraulic power conversion in planar nanochannels,” Phys. Rev. Lett. 103, 144503 (2009).

${ }^{11}$ S. De Luca, B. D. Todd, J. S. Hansen, and P. J. Daivis, "Molecular dynamics study of nanoconfined water flow driven by rotating electric fields under realistic experimental conditions," Langmuir 30, 3095 (2014).

${ }^{12}$ D. J. Evans and W. B. Streett, "Transport properties of homonuclear diatomics II. Dense fluids,” Mol. Phys. 36, 161-176 (1978).

${ }^{13}$ D. J. Evans and G. P. Morriss, Statistical Mechanics of Nonequilibrium Liquids (Academic Press, 1990)

${ }^{14}$ I.-C. Yeh and M. L. Berkowitz, "Dielectric constant of water at high electric fields: Molecular dynamics study," J. Chem. Phys. 110, 7935 (1999).

${ }^{15}$ J. D. Weeks, D. Chandler, and H. C. Andersen, "Role of repulsive forces in determining the equilibrium structure of simple liquids," J. Chem. Phys. 54, 5237-5247 (1971).

${ }^{16} \mathrm{~K}$. Toukan and A. Rahman, "Molecular-dynamics study of atomic motions in water," Phys. Rev. B 31, 2643 (1985).

${ }^{17}$ Y. Wu, H. L. Tepper, and G. A. Voth, "Flexible simple point-charge water model with improved liquid-state properties," J. Chem. Phys. 124, 024503 (2006).

${ }^{18}$ G. Raabe and R. J. Sadus, "Influence of bond flexibility on the vapor-liquid phase equilibria of water," J. Chem. Phys. 126, 044701 (2007).

${ }^{19}$ I. Pasichnyk and B. Dunweg, "Coulomb interactions via local dynamics: A molecular-dynamics algorithm," J. Phys.: Condens. Matter 16, S3999 (2004).

${ }^{20}$ J. S. Hansen, T. B. Schrøder, and J. C. Dyre, "Simplistic Coulomb forces in molecular dynamics: Comparing the wolf and shifted-force approximations," J. Phys. Chem. B 116, 5738 (2012).

${ }^{21}$ H. C. Anderson, "Molecular dynamics simulations at constant pressure and/or temperature," J. Chem. Phys. 72, 2384 (1980).

${ }^{22}$ A. Furukawa and H. Tanaka, "Nonlocal nature of the viscous transport in supercooled liquids: Complex fluid approach to supercooled liquids," Phys. Rev. Lett. 103, 135703 (2009). 